\title{
Comparison of the measured and predicted response of the Earth Radiation Budget Experiment active cavity radiometer during solar observations
}

\author{
J. R. Mahan, N. E. Tira, Robert B. Lee III, and R. J. Keynton
}

\begin{abstract}
The Earth Radiation Budget Experiment consists of an array of radiometric instruments placed in earth orbit by the National Aeronautics and Space Administration to monitor the longwave and visible components of the earth's radiation budget. Presented is a dynamic electrothermal model of the active cavity radiometer used to measure the earth's total radiative exitance. Radiative exchange is modeled using the Monte Carlo method and transient conduction is treated using the finite element method. Also included is the feedback circuit which controls electrical substitution heating of the cavity. The model is shown to accurately predict the dynamic response of the instrument during solar calibration.
\end{abstract}

\section{Introduction}

The climate is ultimately controlled by the distributions of solar radiation absorbed and longwave radiation emitted by the earth and its ocean-atmosphere system. Therefore, if long-term weather and climate patterns eventually are to be understood to the point that they can be predicted, the earth's radiation budget must be accurately monitored. This requirement has led the National Aeronautics and Space Administration (NASA) to propose and carry out the Earth Radiation Budget Experiment (ERBE). A system of three satellites incorporating a combination of proved concepts and new technology has been placed in earth orbit to make detailed earth radiation budget measurements. The three ERBE satellites were launched by the NASA space shuttle on 5 Oct. 1984, 12 Dec. 1984, and 17 Sept. 1986. The ERBE mission objectives have been described by Barkstrom. ${ }^{1}$

ERBE consists of an array of radiometric channels having essentially identical configurations on all three satellites. These include total and short-wavelength, wide and medium field-of-view nonscanning earthviewing channels, and scientifically complementary narrow field-of-view scanning channels, as well as a solar monitor channel.

J.R. Mahan and N. E. Tira are with Virginia Polytechnic Institute \& State University, Mechanical Engineering Department, Blacksburg, Virginia 24061-0238; the other authors are with NASA Langley Research Center, Atmospheric Sciences Division, Hampton, Virginia 23665-5225.

Received 31 March 1988.
The ERBE instruments are designed to measure the emitted and solar reflected components of the earth's radiation, as well as the solar radiant flux incident to the earth, to $<1 \%$ uncertainty over a 2 -yr orbital lifetime. The spatial resolution of these measurements ranges from a $10^{\circ}$ earth central angle for the medium field-of-view channels to full global coverage in the case of the wide field-of-view channels. Temporal sampling is as required by climatologists to generate monthly radiation budget data. The reader is referred to Refs. 1-5 for further descriptions of these instruments.

\section{Radiometer Design and Operation}

An active cavity radiometer (ACR) works by automatically supplying electrical heat to the instrument cavity in just the right amount to ensure that the sum of incident radiation and electrical power is always constant. Then if the radiative input decreases, the electrical heat input increases by the same amount. The output signal is the voltage drop across an electrical heating element in the cavity wall required to maintain this balance.

This paper describes and validates a dynamic thermal model for the total earth-viewing channels of ERBE. The model is suitable for evaluating the channels' performance during steady state operation and for studying the effects of the thermal and electrical time delays. The model is used to simulate a solar calibration, and the response predicted using the mod$\mathrm{el}$ is shown to compare quite favorably with data from actual solar observations.

Solar calibration of the earth-viewing channels on the ERBE satellites was performed weekly during the first month of operation and biweekly thereafter. 
This calibration establishes the accuracy of these radiometer instruments by using them to measure a known quantity, the solar constant. It is also possible to measure the variation of the solar constant itself over a certain scale using the solar monitor.

The ERBE ACR instrument, as configured during solar calibration, is shown in Fig. 1. It consists of an optical system and two sensing elements, one active and the other passive. The optical system is composed of a solar port, a truncated hemispheric field-ofview limiter, and a base plate having a precision aperture at its center. The active and passive sensing elements are similar in that both have a thermal impedance which connects a cavity to a heat sink. The thermal impedance is in the form of a cylindrical sleeve surrounding and concentric with the cavity. In each case, a coupling ring connects the cavity to the thermal impedance.

Each of the cavities is a $30^{\circ}$ cone with a length of $14.94 \mathrm{~mm}$ bonded to a cylinder that has a length of 5.49 $\mathrm{mm}$ and a diameter of $8.0 \mathrm{~mm}$. The thermal impedance sleeve, which is connected to the cavity by a coupling ring, is $23.0 \mathrm{~mm}$ in length and $10.28 \mathrm{~mm}$ in diameter. All four parts are made out of $0.0635-\mathrm{mm}$ thick electrodeposited silver (99.99\% pure).

The surfaces of the cavity (cone and cylinder) which face the opening are coated with a high specular reflective black paint, having a high absorptivity $(\alpha \cong 0.9)$. Windings of electrical heater wire are wrapped around the external surfaces of the cavity. These windings are covered with an aluminized Mylar insulation jacket to minimize radiative exchange between the rearward facing surfaces of the cavity and their surroundings.

The end of the thermal impedance sleeve near its junction with the coupling ring is wound with a platinum wire which acts as a resistance temperature detector (RTD). The windings, along with the external surface of the sleeve, are also covered with an aluminized Mylar insulation jacket.

The purpose of the resistance temperature detector is to indirectly measure heat conduction from the cavity. A temperature change at the RTD, caused by a change in the heat conduction rate from the cavity, is converted by a deflection bridge into an equivalent voltage change. This causes the heater energizing voltage to either increase or decrease so that the temperature drop between the RTD and the heat sink is driven back to its set point value, regardless of the amount of radiation input. An equivalent version of the circuit which implements this feedback function is shown in Fig. 2.

The radiation entering the active cavity is implied based on the assumption of exact instantaneous equivalence between radiative and electrical heat input. That is, the temperature difference between the active sensing element RTD and the heat sink is assumed to respond instantaneously to changes in cavity heating and to be the same for a given amount of heat input whether this input is electrical or radiative. The RTD on the reference sensing element is maintained at a constant temperature while the RTD on the active

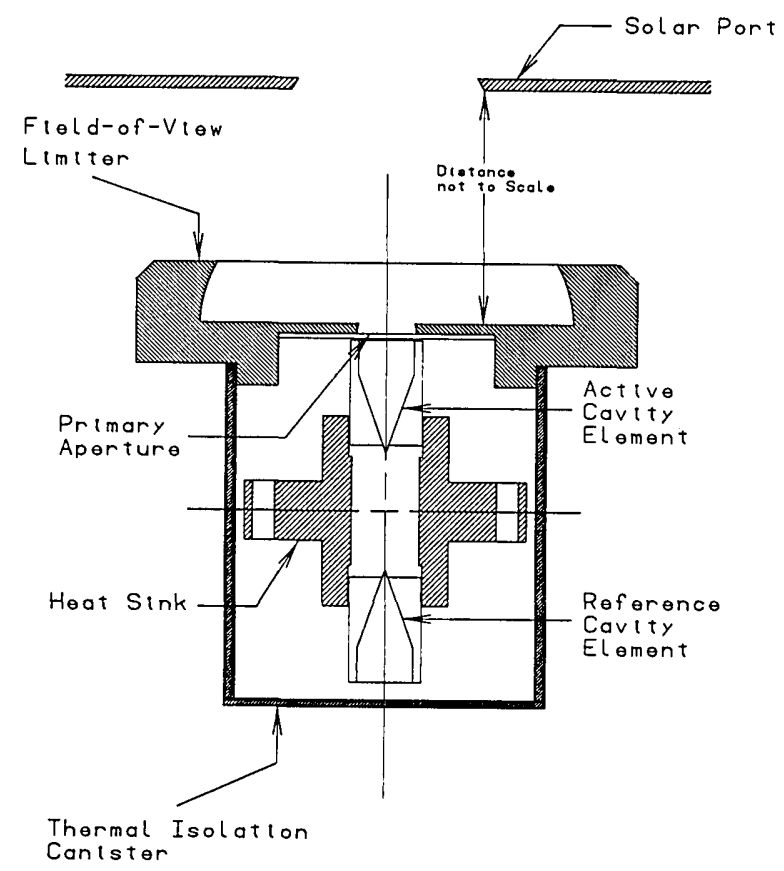

Fig. 1. ERBE total, wide field-of-view radiometer configured for solar calibration.

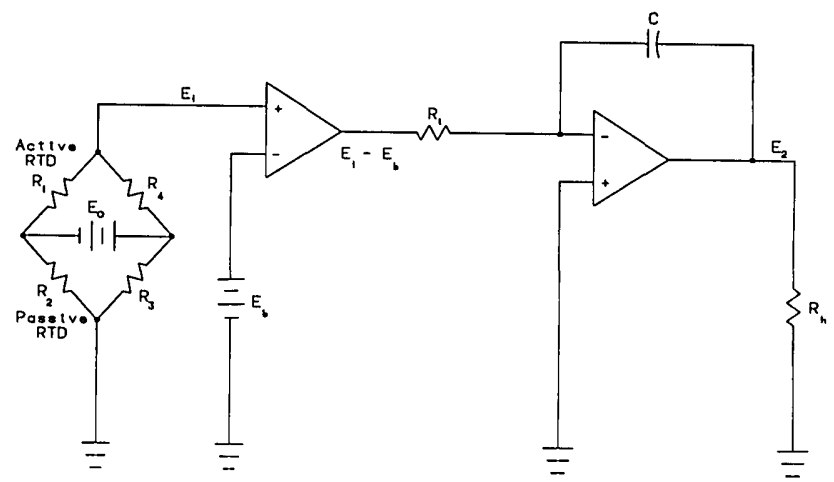

Fig. 2. Electrical substitution heater feedback control circuit.

cavity is maintained at a slightly higher temperature so that the bridge circuit always operates in the deflected mode. The somewhat simplified equivalent circuit of Fig. 2 uses as inputs the resistances $R_{1}$ and $R_{2}$ of the RTDs located on the thermal impedance sleeves of the active and reference sensors; respectively. The detector output is a digitized (13-bit) direct measurement of the active cavity heater voltage, which ranges from 0 to $10 \mathrm{~V}$. The mathematical description of the operation of the electrical circuit which implements this control function is described later in this paper.

\section{Modeling Tools}

Two tasks must be undertaken to formulate an unsteady thermal model of the Earth Radiation Budget Experiment active cavity radiometer. First, the radiative exchange inside the cavity must be accounted for and, second, the unsteady heat conduction in the cavi- 
ty walls and along the thermal impedance must be described.

\section{A. Radiative Exchange in the Radiometer Cavity and with} Its Surroundings

The accurate measurement of radiative energy fluxes using active cavity radiometers requires that the instrument cavity have an apparent absorptivity very close to unity $\left(\alpha_{a} \cong 1.0\right)$. The apparent absorptivity of an isothermal cavity is equal to its apparent emissivity, and this latter is defined as the ratio of the power emitted by the cavity to the power emitted by a black surface of the same temperature stretched across the cavity opening. Although only black (ideal) surfaces have an absorptivity of unity, a cavity consisting of a cylindrical tube terminated by a cone can have an apparent absorptivity closely approaching unity if the cavity walls themselves are highly absorptive and primarily specular (mirrorlike) reflectors. The shape of the ERBE ACR cavity has been conceived to ensure that the radiation incident to the opening suffers an average of six specular reflections before it escapes through the opening.

The Monte Carlo method is used to calculate the radiation distribution factors needed to perform the radiative heat transfer analysis. To implement the Monte Carlo method in the present model, both the cylindrical and conical parts of the radiometer cavity are divided into surface elements whose areas are equal on each part. The distribution factor $D_{j}^{\theta}$ for collimated solar radiation incident to the aperture at an angle of incidence $\theta$ is defined as the fraction of the solar energy entering the cavity at that angle which is absorbed by surface element $j$. Because of the high absorptivity of the cavity, this portion of energy is due mainly to direct radiation for directly illuminated elements. However, it also includes radiation reflected from all cavity elements including the one in question.

If $H$ is the flux in $\mathrm{W} / \mathrm{m}^{2}$ falling on a surface normal to the collimated beam, $A_{i}^{p}$ is the area of element $i$ normal to the collimated beam in $\mathrm{m}^{2}$, and $\theta$ is the angle of incidence of the beam with respect to the aperture normal, the energy absorbed by element $j$ due to this collimated beam is

$$
Q_{j}^{\theta}=H D_{j}^{\theta} \sum_{i=1}^{N} A_{i}^{p} .
$$

In Eq. (1), $N$ is the number of directly illuminated elements.

Collimated radiation may be thought of as being composed of an infinite number of parallel rays that are uniformly distributed over the illuminated area. However, the analysis in this paper assumes that the effect of the rays incident to a given element can be represented by a single vector of equivalent strength incident at its centroid. Therefore, the collimated radiation is represented by a finite number $N$ of vectors incident to the centroids of the $N$ elements illuminated at a given angle of incidence. Of course, $N$ depends on the number of elements into which the cavity has been divided as well as the angle of the

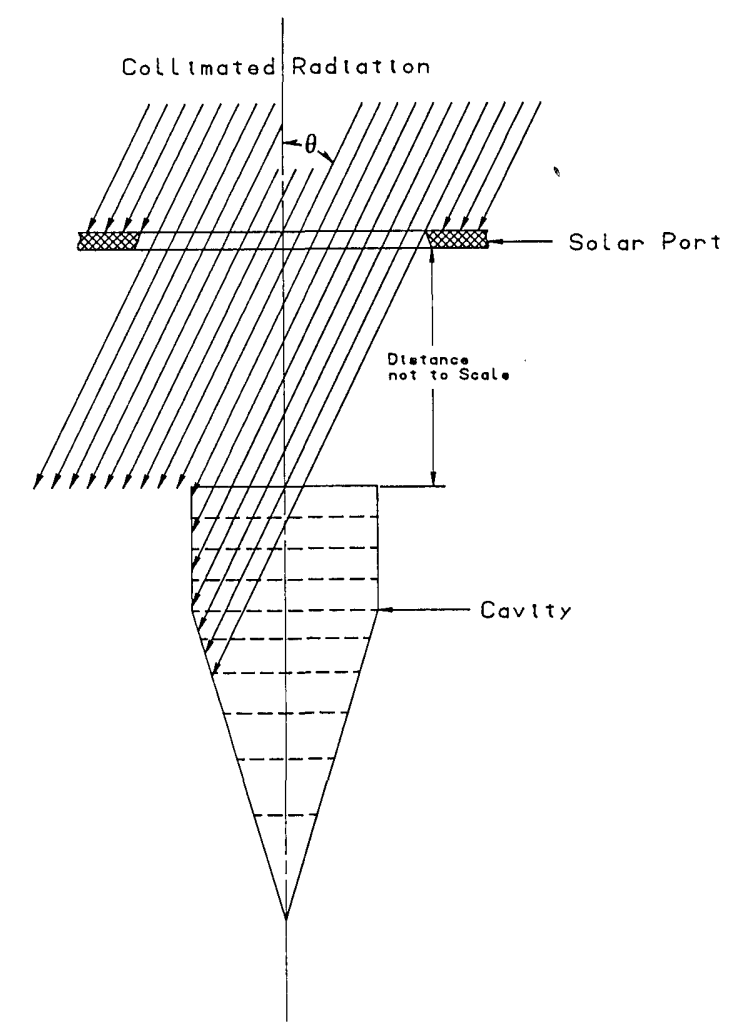

Fig. 3. Collimated solar radiation entering cavity through solar port.

incident collimated beam. It is important to note that the validity of this model increases as the number of cavity elements is increased; indeed, the guiding principle rigorously followed in this work is that the analysis should become exact in the limit as the number of elements becomes infinite.

Collimated radiation entering the cavity during solar calibration must pass through the solar port, as shown in Fig. 3. Therefore, the solar port can be thought of as the source of a beam of collimated radiation. The source is composed of a certain number of source points in the plane of the solar port whose number corresponds to the number of illuminated cavity elements. The number and locations of the source points are easily calculated using the positions of the cavity element centroids and the angle of incidence of the beam on the precision aperture. It is important to note that a cavity element is considered illuminated if its centroid can be seen through the aperture and the solar port at a given viewing angle $\theta$. Some of the elements whose centroids are not seen may be partially illuminated, and some of the elements whose centroids are seen may not be completely illuminated. The errors associated with this effect tend to average out, becoming negligible as the number of cavity elements becomes large.

The Monte Carlo method used in the calculation of the distribution factors is based on two assumptions: (1) radiative interchange among the cavity elements can be simulated by tracing a large number of discrete energy bundles, and (2) the disposition of a given ener- 
gy bundle when it arrives at an element is determined by interpreting the surface properties in terms of the laws of probability.

Calculation of the distribution factors is performed by following a large number of energy bundles entering the cavity from a specified source point in the plane of the solar port. With each incidence, these energy bundles are either absorbed or reflected by the cavity surfaces. Most of the energy bundles are eventually absorbed, with the balance escaping through the aperture. The source of the energy bundles and the direction of their original path are both known, as is the point of incidence (the centroid) on an element of the cavity walls for the original path. The remainder of the path depends on the surface properties and the statistical nature of the Monte Carlo method.

The distribution factors for collimated radiation incident to the cavity are then given by

$$
D_{j}^{\theta}=\frac{\sum_{i=1}^{N}\left(A_{i}^{p} \frac{K_{i j}}{K_{i}}\right)}{\sum_{i=1}^{N} A_{i}^{p}},
$$

where $A_{i}^{p}$ is the area of cavity element $i$ projected along the angle of incidence $\theta, K_{i}$ is the number of energy bundles entering the solar port and incident on the centroid of element $i, K_{i j}$ is the number of these energy bundles ultimately absorbed by element $j$, and $N$ is the total number of illuminated elements.

\section{B. Finite Element Method Applied to the Transient Conduction Problem}

The continuum problem of heat conduction in the cavity model can be solved using any of several discretization methods. Theoretically, for any continuum problem a solution may be obtainable for formal mathematical manipulations. However, these manipulations often require simplifications that may render the solution inaccurate, especially when geometric complications are involved. In these cases, it is necessary to resort to a numerical method such as the finite element method.

The ACR sensing element is geometrically 3-D; however, it can be modeled as a 2-D thermal domain. This is because it is made of a very thin silver sheet whose thermal conductivity is very high, thus precluding the possibility of temperature differences through the wall thickness. The heat conduction equation for such a domain is given by

$$
\frac{\partial}{\partial x}\left(k \frac{\partial T}{\partial x}\right)+\frac{\partial}{\partial y}\left(k \frac{\partial T}{\partial y}\right)+G=\rho c \frac{\partial T}{\partial t},
$$

where $k$ is the thermal conductivity, $\rho$ is the mass density, $c$ is the specific heat, and $G$ is a volumetric heat source term which includes both locally absorbed radiation and the electrical heat source. The details of the finite element solution of Eq. (6) are given in Ref. 6.

\section{Model Formulation}

The unsteady thermal model of the active sensing element is formulated in this section. The model includes the thermal impedance, the cavity and its electrical heater, and the feedback circuit. Aspects of the radiometer optical system are also considered in this analysis. The precision aperture is assumed to fit flush with the cavity opening. Furthermore, there is no heat exchange between the precision aperture and the cavity, or between the solar port and the cavity. Therefore, the only role the solar port plays is to limit the angle of incidence and, therefore, the shape of the collimated beam entering the cavity through the precision aperture.

Radiative input can be any one or a combination of the following types: (1) uniform collimated radiation, (2) a diffuse source field, and (3) a single narrow beam. Each of these types of radiation is incident on the inside surface of the cavity shell through the precision aperture. Radiative interchange is allowed inside the cavity and between the cavity and its surroundings in front of the aperture. Radiative exchange among the thermal impedance, the outside surface of the cavity, and the heat sink is assumed negligible. The validity of this assumption is based on two practical design features: the presence of the aluminized Mylar jackets insulating and shielding the outside surface of the cavity and the thermal impedance from their surroundings, and the small temperature difference between the cavity and the other components of the radiometer $(<1 \mathrm{~K})$.

\section{A. Radiative Heat Input}

The surfaces of the active cavity radiometer (ACR) are assumed to behave like diffuse emitters and diffuse-specular reflectors. The kinds of radiation input that are considered in this analysis require three types of distribution factor:

(1) $D_{j}^{\theta}$, defined as the fraction of energy absorbed by element $j$ due to a collimated beam entering the cavity at angle $\theta$. This fraction of energy includes both direct radiation and all possible diffuse and specular reflections.

(2) $D_{i j}$, defined as the fraction of energy diffusely emitted by element $i$ which is absorbed by element $j$, including both direct radiation and all possible diffuse and specular reflections.

(3) $D_{j(l, m)}$, defined as the fraction of energy contained in a single narrow beam entering the cavity along a vector $(\mathbf{l}, \mathrm{m})$ which is absorbed by element $j$. The vector (l,m) represents the direction of the narrow beam going from an aperture point denoted by $l$ to a cavity element centroid denoted by $m$. Again, this fraction of energy includes both direct radiation and all possible diffuse and specular reflections.

The method for computing the distribution factors $D_{j}^{\theta}$ has already been described, and the detailed description of their calculation, including computer codes, is given in Ref. 6. The other two types of distribution factor are calculated in a similar manner, as described in Ref. 7. 
Each element $j$ in the cavity is subject to a net radiative exchange that is equal to the difference between the energy absorbed, $Q_{\text {rad }, j}^{\text {in }}$, and the energy emitted, $Q_{\mathrm{rad}, j}^{\text {out }}$, by that element, or

$$
Q_{\mathrm{rad}, j}=Q_{\mathrm{rad}, j}^{\text {in }}-Q_{\mathrm{rad}, j \cdot}^{\text {out }}
$$

The absorbed energy, $Q_{\mathrm{rad}, j}^{\mathrm{in}}$, is composed of any one or a combination of the three radiation input types described earlier. It also includes radiation absorbed from all the cavity elements including element $j$. Considering the case where all possible radiative input types are present, $Q_{\mathrm{rad}, j}^{\mathrm{in}}$ is

$$
Q_{\mathrm{rad}, j}^{\mathrm{in}}=Q_{C B, j}+Q_{c a v, j}+Q_{S F, j}+Q_{V, j}
$$

where $Q_{C B, j}$ is the energy absorbed due to a collimated beam,

$Q_{\mathrm{cav}, j}$ is the energy absorbed from all cavity elements,

$Q_{S F, j}$ is the energy absorbed due to the uniform diffuse source field, and

$Q_{V, j}$ is the energy absorbed due to a single narrow beam.

The energy absorbed by element $j$ due to collimated radiation was given in Eq. (1) and is rewritten here as

$$
Q_{C B, j}=H D_{j}^{\theta} \sum_{k=1}^{N} A_{k}^{p},
$$

where $N$ is the number of illuminated elements, $A_{k}^{p}$ is the area of element $k$ normal to the collimated beam, $H$ is the collimated beam flux and $D_{j}^{\theta}$ is the distribution factor associated with element $j$ and a collimated beam incident to the aperture at angle $\theta$.

The energy emitted by all cavity elements that is absorbed by element $j$ is given by

$$
Q_{\mathrm{cav}, j}=\sum_{i=1}^{n} \varepsilon A_{i} \sigma T_{i}^{4} D_{i j} \text {, }
$$

where $n$ is the total number of cavity elements, $\varepsilon$ is the total hemispheric emissivity (assumed to be the same for all elements), $A_{i}$ is the surface area of element $i, \sigma$ is the Stefan-Boltzmann constant, $T_{i}$ is the average temperature of element $i$, and $D_{i j}$ is the appropriate distribution factor.

A special form of the principle of reciprocity is applicable to the distribution factors; that is,

$$
\varepsilon_{i} A_{i} D_{i j}=\varepsilon_{j} A_{j} D_{j i} .
$$

Now since $\varepsilon_{i}$ and $\varepsilon_{j}$ are equal in this analysis, they can be divided out in Eq. (8). The resulting equation is then substituted into Eq. (7) and the expression for $Q_{\text {cav }, j}$ then becomes

$$
Q_{\mathrm{cav}, j}=\sum_{i=1}^{n} \varepsilon A_{j} \sigma T_{i}^{4} D_{j i}
$$

The energy incident on element $j$ due to the uniform diffuse source field is given by

$$
Q_{S F, j}=\varepsilon A_{j} \frac{F}{A_{a}} D_{j a},
$$

where $A_{a}$ is the area of the aperture and $F$ is the strength of the source field.
The last term of Eq. (7), $Q_{V, j}$, is given by

$$
Q_{V, j}=V D_{j(1, \mathrm{~m})} \text {, }
$$

where $V$ is the magnitude of the single vector $(1, \mathbf{m})$ and $D_{j(1, \mathrm{~m})}$ is the distribution factor associated with vector $(\mathbf{l}, \mathbf{m})$ and element $j$. Substituting Eqs. (6) and (9)(11) into Eq. (5), the combined radiative input absorbed by element $j$ becomes

$$
Q_{\mathrm{rad}, j}^{\mathrm{in}}=H D_{j}^{\theta} \sum_{k=1}^{N} A_{k}^{p}+\sum_{i=1}^{n} \varepsilon A_{j} \sigma T_{i}^{4} D_{j i}+\varepsilon A_{j} \frac{F}{A_{a}} D_{j a}+V D_{j(1, \mathrm{~m})} .
$$

Of course, element $j$ emits radiation to its surroundings according to the Stefan-Boltzmann law,

$$
Q_{\mathrm{rad}, j}^{\mathrm{out}}={ }_{\varepsilon} A_{j} \sigma T_{j}^{4} .
$$

Now the net radiative exchange between element $j$ and its surroundings can be formulated by substituting Eqs. (12) and (13) into Eq. (4), yielding

$$
\begin{aligned}
Q_{\mathrm{rad}, j}= & H D_{j}^{\theta} \sum_{k=1}^{N} A_{k}^{p}+\sum_{i=1}^{n} \varepsilon A_{j} \sigma T_{i}^{4} D_{j i} \\
& +\varepsilon A_{j} \frac{F}{A_{a}} D_{j a}+V D_{j(1, \mathrm{~m})}-\varepsilon A_{j} \sigma T_{j}^{4} .
\end{aligned}
$$

Finally, the equivalent volumetric heat generation is obtained by dividing Eq. (14) by the volume of element $j, V_{j}=A_{j} \delta$, where $\delta$ is the element thickness:

$$
\begin{aligned}
G_{\mathrm{rad}, j}= & \frac{1}{A_{j} \delta} H D_{j}^{\theta} \sum_{k=1}^{N} A_{k}^{p}+\frac{1}{\delta} \sum_{i=1}^{n} \varepsilon \sigma T_{i}^{4} D_{j i} \\
& +\frac{1}{A_{a} \delta} \varepsilon F D_{j a}+\frac{1}{A_{j} \delta} V D_{j(1, \mathrm{~m})}-\frac{1}{\delta} \varepsilon \sigma T_{j}^{4} .
\end{aligned}
$$

\section{B. Electrical Heat Input}

The electrical heater is a fine wire wound around the outside surface of the cavity. It is assumed that heat generation in the heater wire is completely transferred to the cavity wall. The resistance temperature detector (RTD), also a fine wire, is wound around the thermal impedance sleeve near its junction with the coupling ring. Two of the arms of the deflection bridge in Fig. 2 are fixed resistors $\left(R_{3}\right.$ and $\left.R_{4}\right)$. A third arm is the RTD associated with the reference cavity $\left(R_{2}\right)$. This cavity, and thus the RTD, is precisely controlled to a constant temperature near that of the heat sink during earth viewing. During calibration against the internal blackbody radiation standard and during solar calibration, the temperature of the reference cavity, and thus the reference RTD, is raised to offset the correspondingly larger heat fluxes incident to the active cavity. Therefore, when simulating solar calibration, the resistance of the reference RTD is held constant. For simplification, the resistance of this arm of the bridge is assumed to be the same as that of the two fixed resistors. This convenient simplification is electrically equivalent to changing the bias voltage $E_{b}$ in Fig. 2 . But $E_{b}$ is an arbitrary component of the calibration constant whose value is to be determined by calibration. Therefore, this simplification in no way compromises the simulation. 
The temperature of the fourth arm, which is that of the active cavity RTD $\left(R_{1}\right)$, is not uniform around the thermal impedance since the cavity temperature itself is not completely uniform. However, the cavity temperature nonuniformity is $<1 \mathrm{~K}$, and the temperature variation at the location of the RTD would be even smaller. Consequently, the RTD may be assumed to take the average temperature of the line of nodes which it covers.

Ohm's law requires that the output voltage of the deflection bridge be given by (refer to Fig. 2)

$$
E_{1}=E_{0}\left(\frac{R_{1}}{R_{1}+R_{4}}-\frac{R_{2}}{R_{2}+R_{3}}\right) \text {, }
$$

where $E_{0}$ is the bridge supply voltage, $R_{1}$ is the active RTD resistance, $R_{2}$ is the passive RTD resistance, and $R_{3}$ and $R_{4}$ are the other bridge arm fixed resistances.

At the equilibrium condition

$$
R_{1}=R+\Delta R_{1} \text {, }
$$

where $R$ is the resistance of the active cavity RTD which would produce a zero bridge deflection. The quantity $\Delta R_{1}$ is always greater than zero because the temperature of the active RTD is controlled to be higher than that of the reference RTD. When the amount of radiation incident to the cavity changes, the cavity adopts a new temperature distribution, and the average temperature of the RTD changes accordingly. This, in turn, causes the value of $\Delta R_{1}$ to change. The other resistances are maintained constant at $R$. Substituting Eq. (17) into Eq. (16) and taking into consideration the small value of $\Delta R_{1}$, which permits the result to be linearized, the expression for the output voltage becomes

$$
E_{1}=\frac{E_{0}}{4}\left(\frac{R_{1}}{R}-1\right) .
$$

The resistance of the RTD is assumed to vary linearly with temperature; therefore, it can be expressed by

$$
R_{1}=R\left[1+\alpha\left(T_{1}-T_{h s}\right)\right],
$$

where $\alpha$ is the resistance temperature coefficient of the RTD and $T_{1}$ and $T_{h s}$ are the RTD and the heat sink temperatures, respectively. Substitution of Eq. (19) into Eq. (18) gives the output voltage in terms of the temperatures.

$$
E_{1}=\frac{\alpha}{4} E_{0}\left(T_{1}-T_{h s}\right) .
$$

The input voltage to the circuit integrator is the difference between the output voltage of the bridge $E_{1}$ and the bias voltage $E_{b}$. The integrator processes this voltage difference to give an output voltage $E_{2}$, which is the energizing voltage for the electric heater wrapped around the active cavity. The output voltage of the circuit as a function of time can be expressed by the differential equation

$$
\frac{d E_{2}}{d t}=\frac{E_{b}-E_{1}}{\tau},
$$

where $\tau$ is the electrical time constant of the integrator,
$\tau=R_{i} C$ (see Fig. 2). On substitution for $E_{1}$ from Eq. (20), Eq. (21) becomes

$$
\frac{d E_{2}}{d t}=\frac{E_{b}}{\tau}-\frac{\alpha}{4 \tau} E_{0}\left(T_{1}-T_{h s}\right) .
$$

Noting that the bias voltage is given by

$$
E_{b}=\frac{\alpha}{4} E_{0} \Delta T,
$$

the differential equation for $E_{2}$ can finally be written as

$$
\frac{d E_{2}}{d t}=\frac{\alpha}{4 \tau} E_{0}\left[\Delta T+\left(T_{h s}-T_{1}\right)\right] .
$$

The finite difference method is used to determine the relationship between the values of $E_{2}$ at two consecutive time steps $p$ and $p+1$; that is,

$$
E_{2}^{p+1}=E_{2}^{p}+\Delta t \frac{\alpha}{4 \tau} E_{0}\left[\Delta T+\left(T_{h s}-T_{1}\right)\right] .
$$

The heater power output $Q_{\text {elec }}$ is given by

$$
Q_{\text {elec }}=\frac{E_{2}^{2}}{R_{h w}},
$$

where $R_{h w}$ is the resistance of the heater wire (1398.1 $\Omega)$.

The volumetric heat generation in each surface element covered by the heater wire is given by

$$
G_{\text {elec }, j}=\frac{Q_{\text {elec }}}{A_{h w} \delta},
$$

where $A_{h w}$ is the total area of the cavity covered by the heater wire and $\delta$ is the thickness of the cavity wall. In terms of the heater voltage $G_{\text {elec }, j}$ is expressed by

$$
G_{\text {elec }, j}=\frac{E_{2}^{2}}{R_{h w} A_{h w} \delta} .
$$

Finally, an expression for the volumetric heat generation at time step $p+1$ may be obtained by substituting Eq. (25) into Eq. (28), giving

$$
G_{\text {elec }, j}^{p+1}=\frac{1}{R_{h w} A_{h w^{\delta}}}\left\{E_{2}^{p}+\Delta t \frac{\alpha}{4 \tau} E_{0}\left[\Delta T+\left(T_{h s}-T_{1}\right)\right]\right\}^{2} .
$$

\section{Results and Discussion}

All simulations have been performed using a finite element mesh consisting of ten circumferential divisions around the cavity, four axial divisions along the cylinder, six axial divisions along the cone, one radial division on the coupling ring, and four axial divisions along the thermal impedance. This results in a 620node conduction analysis mesh. The geometry of the radiometer is that of the ERBS total wide field-of-view channel, whose dimensions have already been given. The solar port has a diameter of $17.42 \mathrm{~mm}$ and it is $76.76 \mathrm{~mm}$ in front of the precision aperture. The cavity is made of pure silver having a thermal conductivity of $429.0 \mathrm{~W} / \mathrm{m} \mathrm{K}$, a specific heat of $235 \mathrm{~J} / \mathrm{kg} \mathrm{K}$, and a mass density of $10,500 \mathrm{~kg} / \mathrm{m}^{3}$. The heat sink temperature is taken to be constant at $307 \mathrm{~K}$ throughout the analysis. The linear temperature coefficient of 
resistance $\alpha$ for the resistance temperature detector is taken to be $0.0016(1 / \mathrm{K})$.

The set point temperature drop between the active cavity RTD and the heat sink is found to be $\sim 0.78 \mathrm{~K}$ based on a steady state analysis of the thermal model.

The simulation of the solar calibration event is based on a transient radiative heat input in the form of a collimated beam incident at a series of angles. This beam sweeps over the radiometer aperture from an angle of 8.8 to an angle of $-8.8^{\circ}$ with respect to the aperture normal. This angle range is determined by the diameters of the solar port and the aperture and by the distance between them, and includes all angles which allow solar radiation to enter the cavity. Figure 3 shows this geometry but not to scale.

\section{A. Results for the Distribution Factor Calculation for Collimated Radiation}

The codes developed during this effort have the capability of computing the distribution factors for one collimated beam at a given angle of incidence, or for a family of several collimated beams with a specified angle range and a number of specified angle steps. The distribution factors for the present analysis have been computed for angles ranging between 8.8 and $0.0^{\circ}$; that is, the half-span of angles needed for solar calibration simulation of the ERBS total wide field-ofview channel. Only the half-span of angles is needed because of the symmetry of the cavity and the collimated solar radiation input function: the same distribution factors transformed to the opposite side of the cavity are used for the second half of the calibration. The number of angle steps between 0 and $8.8^{\circ}$ was chosen as thirty-two. This arbitrary choice permits the calculation of the distribution factors at thirtythree angles of incidence. Then taking symmetry into account, there are sixty-four angular increments for a complete solar calibration.

The distribution factors have been calculated using 10,000 energy bundles in the Monte Carlo method. Intuitively, it might at first seem that the accuracy of the distribution factors for collimated radiation, or the other types of radiation, should improve with an increase in the number of energy bundles. However, Mahan et al. ${ }^{8}$ have established that the distribution factor accuracy is also sensitive to the number of elements into which the cavity is divided, and that for a given number of elements there is actually an optimum number of energy bundles. The choice of 10,000 is made based on the findings of that study.

The distribution factors for angles of 7.7, 5.5, 3.3, and $1.1^{\circ}$ are shown in Fig. 4. The vertical axis on the 3$D$ plots represents the magnitudes of the distribution factors. When studying Fig. 4 , it should be recalled that the distribution factor represents the fraction of the solar radiation entering the cavity which is absorbed by a given surface element.

The secondary ridge on the cone in Fig. 4(a) may be attributed to reflected solar radiation. Most other distribution factor plots also show secondary and, in some cases, even higher-order reflections. Also, exam-
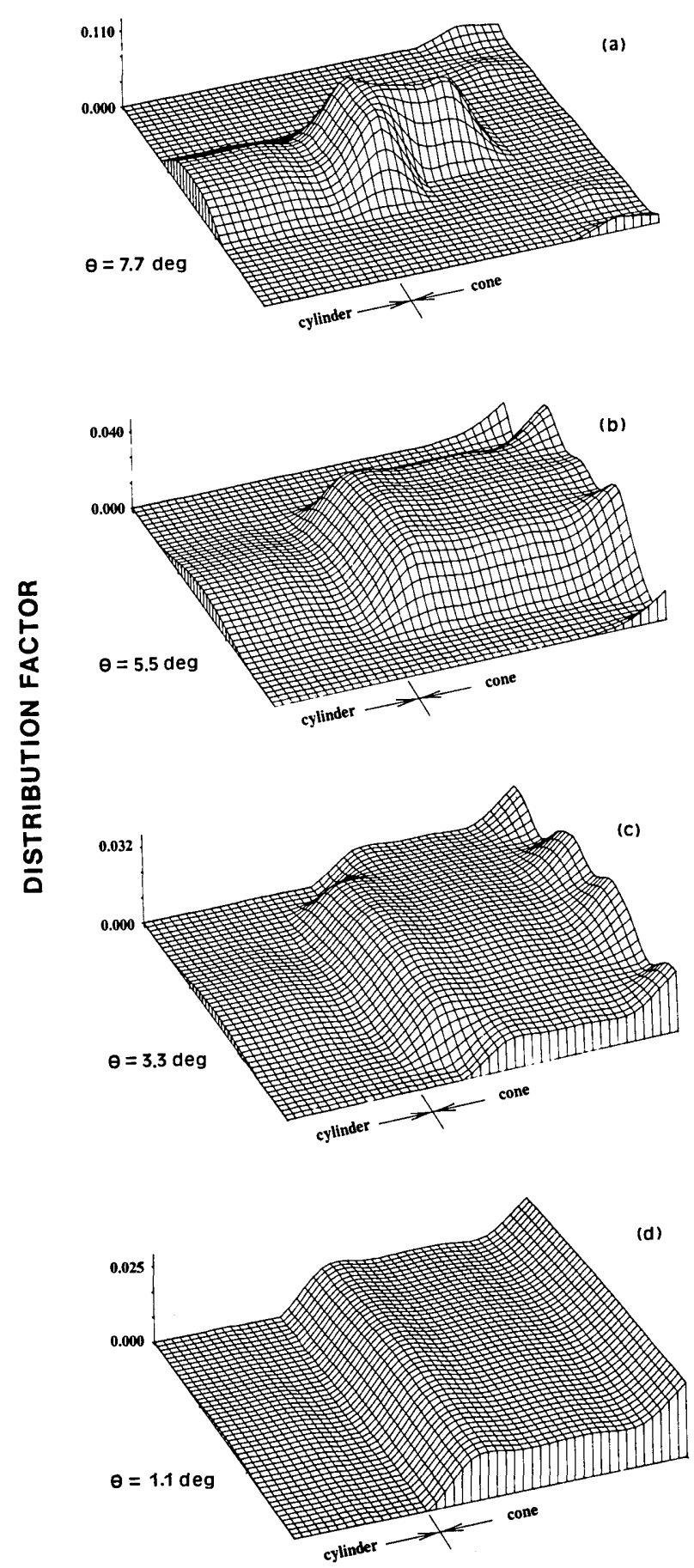

Fig. 4. Radiation distribution factors for a collimated beam incident at angles of (a) $7.7^{\circ}$, (b) $5.5^{\circ}$, (c) $3.3^{\circ}$, and (d) $1.1^{\circ}$.

ination of Fig. 4 reveals that the magnitude of the distribution factors on the directly illuminated cylinder elements is generally less than that on the directly illuminated cone elements. This difference may be explained in terms of the angle between the element surface normal and the collimated beam, which is smaller for a cone element than for a cylinder element.

The higher magnitude of the distribution factors at the cone tip, especially in Figs. 4(b)-(d), is due to the 
fact that only $90 \%$ of the incident radiation is absorbed at its first incidence on the directly illuminated elements. The rest, $10 \%$ is reflected farther down into the cavity. This reflected radiation tends to gather at the cone tip.

\section{B. Results of the Thermal Model}

\section{Performance and Accuracy of the Model}

The model accuracy can be evaluated based on an energy balance on the sensing element, which requires that heat input to the system either be conducted away to the heat sink or emitted out of the cavity aperture; i.e.,

$$
Q_{\text {elec }}^{\text {in }}+Q_{\text {rad }}^{\text {in }}=Q_{\text {emit }}^{\text {out }}+Q_{\text {cond }}^{\text {out }} .
$$

The energy balance error, in percent, is defined as

$$
\text { err }=\left(1-Q_{\text {out }} / Q_{\text {in }}\right) \times 100 \% \text {, }
$$

where $Q_{\text {in }}=Q_{\text {elec }}^{\text {in }}+Q_{\text {rad }}^{\text {in }}$ and $Q_{\text {out }}=Q_{\text {emit }}^{\text {out }}+Q_{\text {cond }}^{\text {out }}$.

The finite element method gives an approximation to the exact solution of an analytical system. The accuracy of this approximation depends on the number and location of the elements in the mesh, among other factors. Therefore, a study of the effects of varying the finite element mesh was conducted. This study involved a steady state analysis of the model for a diffuse radiative source field having a strength of 50 $\mathrm{mW}$.

The number of significant figures shown for the results in Table I in many cases exceeds the number that can be justified on the basis of claimed model accuracy. Nonetheless, the excess significant figures are meaningful in the context of comparing similar values within the same numerical model. For example, $Q_{\text {emit }}^{\text {out }}$ and $Q_{\text {cond }}^{\text {out }}$ in Table I are both given to six significant figures even though these quantities could never be measured or calculated to this absolute accuracy. However, the numbers do provide a valid measure of the sensitivity of the results calculated to the parameters of the model.

Table I gives the results for eight steady state cases representing a range of heating scenarios, cavity divisions, and surface properties. The first four cases involve a mesh of $14 \times(2+3)=70$; that is, fourteen circumferential divisions, two axial divisions on the cylinder and three on the cone, totaling 70 elements in the cavity. The last four cases involve a mesh of $10 \times$ $(4+6)=100$. In all cases, the reflectivity ratio $\left(R_{r}\right)$ is maintained at unity while either a $50-\mathrm{mW}$ diffuse radiation heat input or a $50-\mathrm{mW}$ electrical heat input is used with an absorptivity $(\alpha=\varepsilon)$ of either 0.9 or 1.0. The table gives the heat input to the cavity, the radiation emitted and the heat conducted from the cavity, the total heat output from the cavity, and the percent error as defined by Eq. (33).

The percent error for the cases where no radiation is considered (cases 4 and 8 ) is a measure of the accuracy of the finite element conduction model. The results, 0.004 and $0.003 \%$ for the 70 -element mesh and the 100 element mesh, respectively, are inherent errors in the model due to the method of approximation chosen. For each pair of cases with the same conditions ( 1 and 5,2 and 6,3 and 7 , and 4 and 8 ), the 100 -element mesh model produces a percent error which is only slightly lower than that produced by the 70-element mesh model. This verifies that there is no point in increasing the number of elements beyond 100 .

\begin{tabular}{|c|c|c|c|c|c|c|c|c|}
\hline Case \# & Mesh & $\begin{array}{c}Q_{\text {diec }}^{\text {in }} \\
(\mathrm{mW})\end{array}$ & $\begin{array}{c}Q_{\text {rad }}^{\text {in }} \\
(\mathrm{mW})\end{array}$ & $\begin{array}{c}\text { Absorptivity } \\
\alpha=\varepsilon\end{array}$ & $\begin{array}{l}Q_{\text {emt }}^{\text {out }} \\
(\mathrm{mW})\end{array}$ & $\begin{array}{l}Q_{\text {cond }}^{\text {out }} \\
(\mathrm{mW})\end{array}$ & $\begin{array}{c}Q_{\text {emtit }}^{\text {out }}+Q_{\text {cond }}^{o u t} \\
(\mathrm{~mW})\end{array}$ & $\begin{array}{c}\text { Percent }{ }^{1} \\
\text { Error } \\
(\%)\end{array}$ \\
\hline 1 & $14 \times(2+3)$ & 0.0 & 50.0 & 1.0 & 25.6051 & 24.5055 & 50.1106 & -0.221 \\
\hline 2 & $14 \times(2+3)$ & 0.0 & 50.0 & 0.9 & 25.2852 & 24.2006 & 49.4858 & 1.028 \\
\hline 3 & $14 \times(2+3)$ & 50.0 & 0.0 & 0.9 & 25.3077 & 24.7005 & 50.0082 & -0.016 \\
\hline $4^{2}$ & $14 \times(2+3)$ & 50.0 & 0.0 & 0.0 & 0.0 & 49.9978 & 49.9978 & 0.004 \\
\hline 5 & $10 \times(4+6)$ & 0.0 & 50.0 & 1.0 & 25.5572 & 24.4545 & 50.0117 & -0.023 \\
\hline 6 & $10 \times(4+6)$ & 0.0 & 50.0 & 0.9 & 25.5460 & 24.4379 & 49.9839 & 0.032 \\
\hline 7 & $10 \times(4+6)$ & 50.0 & 0.0 & 0.9 & 25.5699 & 24.4346 & 50.0045 & -0.009 \\
\hline $8^{2}$ & $10 \times(4+6)$ & 50.0 & 0.0 & 0.0 & 0.0 & 49.9985 & 49.9985 & 0.003 \\
\hline
\end{tabular}

Table I. Results of Various Tests on the Overall Error and Performance of the Model

1: The percent error is defined as $\left[1-\frac{Q_{\mathrm{emit}}^{\text {out }}+Q_{\mathrm{cond}}^{\text {out }}}{Q_{\text {ln }}}\right] \times 100 \%$,

where $Q_{\text {in }}$ is a $50 \mathrm{~mW}$ radiative heat input from the diffuse source ficld.

2: Radiation is not considered. 
Having determined the error inherent with the finite element conduction analysis, the accuracy of the radiation analysis can now be estimated. The error in the radiation component of the 100 -element model may be estimated by taking the difference in error between any of the cases having both radiation and conduction, for example, cases 5,6 or 7 , and the error of case 8 which involves conduction only. For example, applying this relation to case 7 , the error involved with the out-ofaperture emission portion of the radiative formulation of the model is $0.005 \%$. On the other hand, there is $0.029 \%$ error (case 6 ) involved with the whole formulation of the radiative model for an emissivity of 0.9 , and $0.020 \%$ error for an emissivity of 1.0 (case 5). These errors are mostly due to the Monte Carlo method and its implementation.

In view of the results reported here and in the similar more extensive study in Ref. 6 of the model accuracy, it seems reasonable to claim a conservative model accuracy for equilibrium operation of the ERBE ACR of $0.1 \%$. That is, the predicted and measured instrument responses to a steady input function can be expected to agree to within better than $0.1 \%$.

\section{Evaluation of Equivalence}

Equivalence refers to the ability of the instrument to produce the same temperature at the RTD whether the power added to the cavity is radiative or electrical. Because of its basic importance it needs to be verified for the ERBE ACR. Analytically, nonequivalence, which is the opposite of equivalence, is defined as

$$
\mathrm{NE}=\left(1-Q_{\mathrm{elec}} / Q_{\mathrm{rad}}^{\max }\right) \times 100 \%,
$$

where $Q_{\text {elec }}$ is the electrical substitution heat input which produces the same temperature difference between the resistance temperature detector (RTD) and the heat sink as does $Q_{\mathrm{rad}}^{\max }$ in the absence of electrical substitution heating. Here $Q_{\mathrm{rad}}^{\max }$ is the maximum expected value of incident radiation.

The study of nonequivalence could be performed using any of the three types of radiative input: the collimated radiation, the uniform diffuse source field, or the single narrow beam. However, because the highly nonuniform heating associated with a narrow beam would produce the greatest nonequivalence, this type of heating has been used to establish a conservative estimate of its value.

Figure 5 gives nonequivalence as a function of angle of incidence of a narrow beam entering the cavity. For the majority of the angles of incidence, nonequivalence is of the order of $0.05 \%$. It exceeds $0.07 \%$ only for an angle of over $80^{\circ}$, which is far beyond the maximum angle of incident radiation for the ERBS total wide field-of-view channel permitted by the field-of-view limiter. Consequently, it would not be unreasonable to assume that nonequivalence for collimated radiation in the angle range associated with solar calibration $\left( \pm 8.8^{\circ}\right)$ would be quite a bit lower. Any value below $\pm 0.05 \%$ is, of course, very acceptable given that this is about the accuracy of the model itself for equilibrium operation.

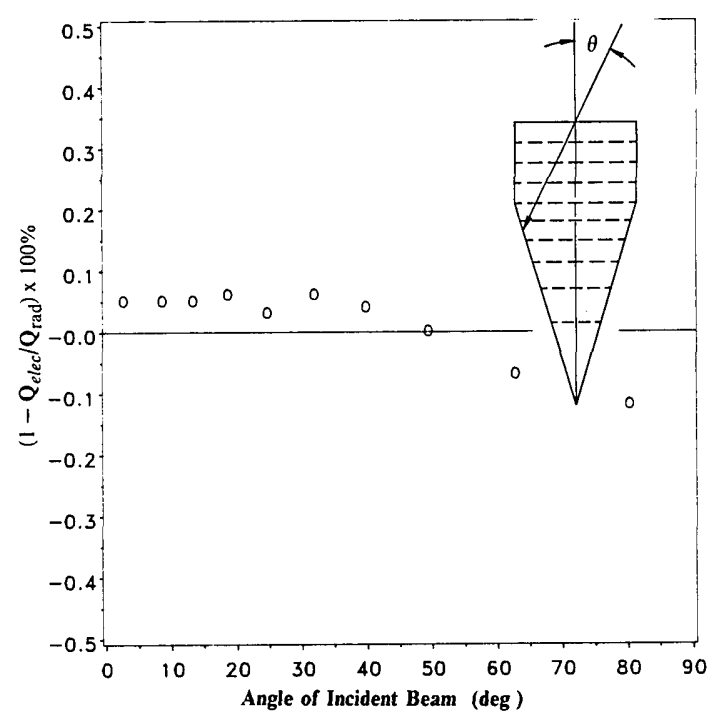

Fig. 5. Nonequivalence for a narrow beam as a function of angle of incidence to the cavity aperture.

The nonequivalence for a diffuse source field has been studied in a different way. At first, only radiative power is input to the model. Then a portion of that power is replaced by electrical heat such that the RTD temperature remains constant. The amount of electrical power needed to produce the same RTD temperature is always within $0.008 \%$ of the radiative power it replaced, indicating excellent equivalence. These results are very important in the sense that they prove that electrical substitution heating is equivalent to within a small error to the radiative heat input when the radiation is from a diffuse source. That is, the very important assumption of equivalence is satisfactorily verified, at least in this special but representative case.

\section{Comparison of Simulated Solar Calibration with the ERBS Data}

The operational ERBS solar calibration data are obtained in the following manner. First, the radiometer instrument is rotated $78^{\circ}$ from its earth-viewing position to its sun-viewing position. The instrument cavity is maintained at a certain temperature level by the electrical heater for a period of time. Then the sun passes in front of the solar port. The ERBS data are arranged in blocks of twenty observations obtained over a period of $16 \mathrm{~s}$. Each block is referred to as a sample, and each observation consists of a count value representing the voltage across the heater. A count value of unity is equivalent to $1 / 819.1 \mathrm{~V}$.

The radiative input to the model during numerical simulation of the solar calibration has been provided in a similar manner. Before the onset of the solar input function, the cavity is given an initial temperature distribution that has been obtained from a steady state solution with electrical heat input only. This produces a thermal state of the model which very closely matches that of the actual instrument just before it is exposed to the sun. 
The choice of the starting time of the calibration event was made by inspection of the ERBS operational data. That is, the event was arbitrarily deemed to begin a few (less than five) observations before the abrupt decrease in the count value associated with the onset of the solar calibration event. The exact starting time of this event was always chosen to maximize the agreement between the simulation and the ERBS data. In this analysis, the highest count value (no sun) is $\sim 7500$, which is equivalent to $60 \mathrm{~mW}$ of electrical heat input. The lowest count value (sun is directly in front of the aperture) is $\sim 3750$, which is equivalent to $15 \mathrm{~mW}$ of electrical heat input.

Two sets of operational data obtained by ERBS are compared with corresponding simulations in this paper. The first set was taken on 28 Dec. 1984, and the second set was taken on 31 Oct. 1985. In each simulation, the solar flux used was equal to the actual solar constant corrected for earth-sun distance. The time periods for these data sets were determined to be 291 and $342 \mathrm{~s}$, respectively, as described above. The model was run using these time periods, an electrical time constant $\tau$ of $0.005 \mathrm{~s}$, a calculation time step of $3.2 \mathrm{~s}$, and a solar input whose distribution on the cavity walls changed in 64 angular increments. The first simulation results are plotted on the same set of axes as the corresponding ERBS data for the 28 Dec. 1984 calibration event in Fig. 6(a). The horizontal line represents the value of the electrical heater input in the absence of solar input $(59.97 \mathrm{~mW})$. The corresponding temperature response of the RTD as determined by the model is shown in Fig. 6(b). This response is slightly above the set point RTD temperature $(307.78 \mathrm{~K}$, represented by the horizontal line) at the beginning of the solar calibration event; i.e., when solar radiation input starts to increase. Around the middle of the event, the RTD response fluctuates around its set point value. When the solar input starts to decrease, the RTD temperature drops below the set point value.

The second simulation result is plotted on the same set of axes as the corresponding ERBS data for the 31 Oct. 1985 calibration event in Fig. 7(a). The corresponding temperature response of the RTD for this case is shown in Fig. 7(b). These results are very similar to those for the $28 \mathrm{Dec}$. 1984 solar calibration. Figures 6(a) and 7(a) show a remarkable agreement between the observed and predicted behavior of the instrument during solar calibration.

\section{Summary and Conclusions}

A dynamic electrothermal model has been formulated for the total, nonscanning channels of NASA's Earth Radiation Budget Experiment. Diffuse-specular thermal radiation exchange is treated by dividing the ACR cavity into 100 surface elements and then using the Monte Carlo method to compute the required radiation distribution factors among these elements. Unsteady heat conduction in the sensing element is treated using a 620-node finite element analysis. As in the actual instrument, the electrical substitution heat input to the cavity is controlled by
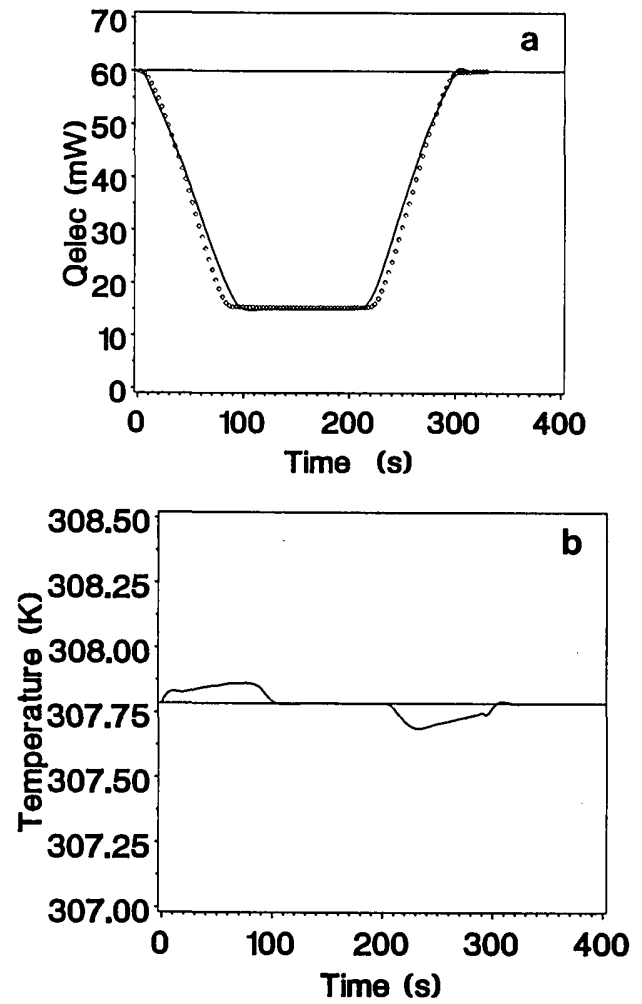

Fig. 6. (a) Comparison of predicted and observed electrical substitution heater power for the solar calibration event of 28 Dec. 1984, and (b) the corresponding variation of predicted RTD temperature with time.
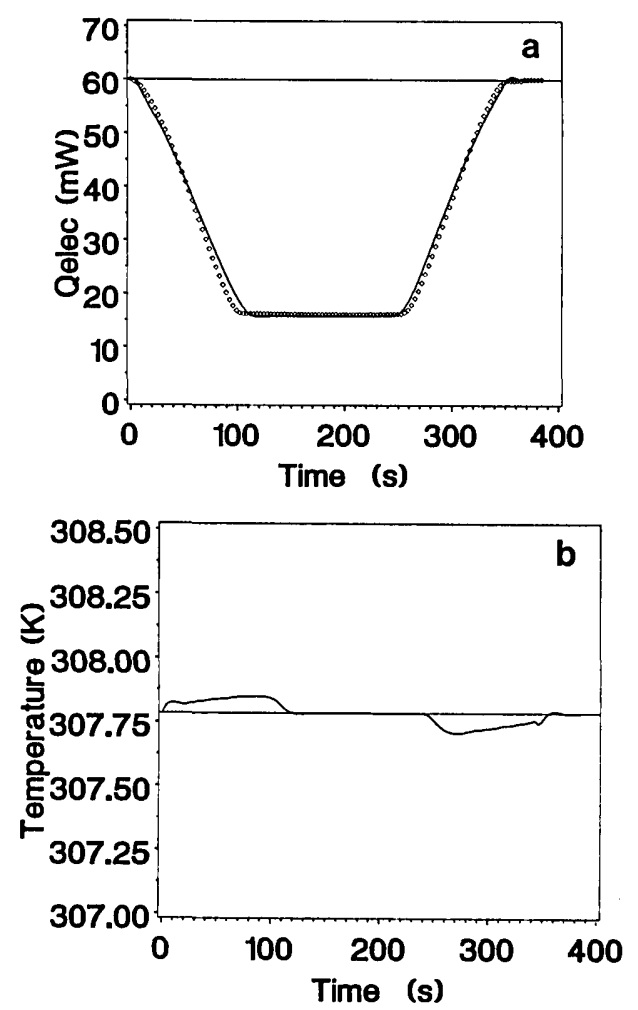

Fig. 7. (a) Comparison of predicted and observed electrical substitution heater power for the solar calibration event of 31 Oct. 1985, and (b) the corresponding variation of predicted RTD temperature with time. 
temperatures calculated on the thermal impedance near its junction with the cavity. A dynamic model of the feedback control circuit is included in the simulation.

The model predicts an equivalence between uniform diffuse radiation input and electrical substitution heat input to the cavity of better than $0.01 \%$. For a single narrow beam radiation input, the maximum nonequivalence is predicted to be $<0.1 \%$; however, the ERBE ACR is never exposed to this extreme form of radiative heating. It may be concluded from this that the maximum predicted nonequivalence is somewhere between 0.01 and $0.1 \%$, but probably nearer to the smaller value. The accuracy of this prediction is itself probably better than $0.1 \%$.

The most important conclusion is that the model correctly simulates the most extreme measurement event the ERBE ACR near experiences, i.e., solar calibration. The extremely good agreement between predicted and observed instrument response demonstrated in this paper suggests that the model could be very useful as a tool for enhanced data reduction. For example, since it performs temporal and spatial integration exactly like the actual instrument, it could possibly be used in the inverse mode to deconvolute operational data. In this same vein, it could be used to test deconvolution models and angularity models for radiative exitance, that is, a sequence of simulated earth scenes could be input into the model and the sensitivity of the instrument output to scene features evaluated.
A debt of gratitude is owed to the Radiation Sciences Branch of the Atmospheric Sciences Division at NASA's Langley Research Center for its support of this work under contract NAS1-18106, task 12.

\section{References}

1. B. R. Barkstrom, "The Earth Radiation Budget Experiment (ERBE)," Bull. Am. Meteorol. Soc. 65, 1170 (1984).

2. R. B. Lee, B. R. Barkstrom, and R. D. Cess, "Characteristics of the Earth Radiation Budget Experiment Solar Monitors," Appl. Opt. 26, 3090 (1987).

3. L. P. Kopia, "The Earth Radiation Budget Experiment Instrument Design Status," in Proceedings, Fourth Conference on Atmospheric Radiation, Toronto (16-18 June 1981).

4. L. P. Kopia, "The Earth Radiation Budget Experiment Scanner Instrument," Rev. Geophys. Space Phys. 24, (1986).

5. M. R. Luther, J. E. Cooper, and G. R. Taylor, "The Earth Radiation Budget Experiment Nonscanner Instrument," Rev. Geophys. Space Phys. 24, 391 (1986).

6. N. E. Tira, "Dynamic Simulation of Solar Calibration of the Total, Earth-Viewing Channel of the Earth Radiation Budget Experiment (ERBE)," M.S. Thesis, Department of Mechanical Engineering, Virginia Polytechnic Institute \& State U., Blacksburg, VA (Dec. 1987).

7. L. D. Eskin, "Application of the Monte Carlo Method to the Transient Thermal Modeling of a Diffuse-Specular Radiometer Cavity," M.S. Thesis, Department of Mechanical Engineering, Virginia Polytechnic Institute \& State U., Blacksburg, VA (1981).

8. J. R. Mahan, F. Kowary, N. Tira, and B. D. Gardiner, "Transient Conduction-Radiation Analysis of an Absolute Active Cavity Radiometer Using Finite Elements," in Proceedings, International Symposium on Thermal Problems in Space-Based Systems, ASME HTD-83, Boston, MA (14-18 Dec. 1987).
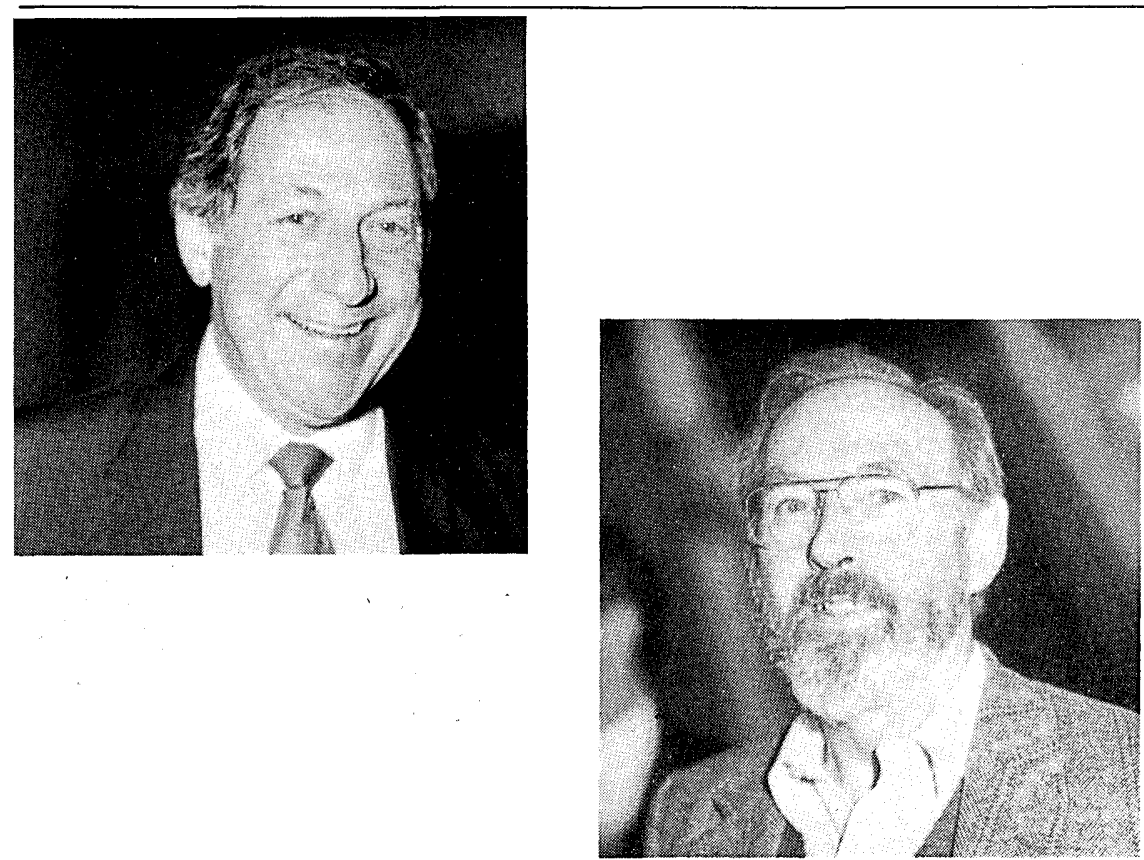

photos:

F. S. Harris, Jr.

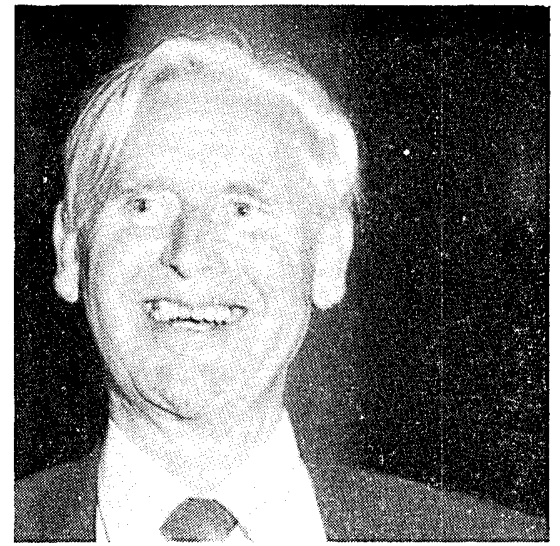

